\section{Protein tyrosine nitration in chronic intramuscular parasitism: immunohistochemical evaluation of relationships between nitration, and fiber type-specific responses to infection}

\author{
Ted H. Elsasser, 1 German Romo, 1 \\ Janet Eastridge,1 Stanislaw Kahl,1 \\ Alfredo Martínez, 3 Changge Feng,2 \\ Cong Jun Li, 1 James L. Sartin, 4 \\ Frank Cuttitta, ${ }^{2}$ Ronald Fayer ${ }^{1}$ \\ 1USDA, Agricultural Research Service, \\ Animal and Natural Resources Institute, \\ Beltsville, MD, USA; \\ ${ }^{2}$ National Institutes of Health, National \\ Cancer Institute, Gaithersburg, MD, USA; \\ 3Oncology Department, Center for \\ Biomedical Research of La Rioja, Spain; \\ 4Department of Anatomy, Physiology and \\ Pharmacology, School of Veterinary \\ Medicine, Auburn University, Auburn, \\ AL, USA
}

\section{Abstract}

The present study was conducted to determine whether preferential muscle catabolism [psoas major $(\mathrm{PM})>$ rectus femoris $(\mathrm{RF})$ ] observed during the chronic intramuscular stage of Sarcocystis cruzi infection could be associated with the pathological consequences of increased protein tyrosine nitration in fibers characteristically more metabolically active due to higher mitochondrial density. Holstein calves were assigned to control (C), or $S$. cruzi-infected (I) groups, $\mathrm{n}=5 /$ group. Calves were euthanized on day 63 of infection. Samples of RF and PM were prepared for metabolic fiber typing (MFT: slow oxidative, SO Type I; fast oxidative glycolytic, FOG - Type IIa; fast glycolytic, FG - Type IIb), fiber area, and immunohistochemical localization of fast myosin heavy chain $2 \mathrm{a}$ and $2 \mathrm{~b}$, nitrotyrosine (NT), and mitochondrial Complex V ATP-synthase. MFT analysis documented that PM contained twice the number of SO fibers compared to RF (32 v 16\%, P<0.002). S0 and F0G fibers (Both higher in mitochondrial density than FG fibers) in both PM and RF were significantly smaller in area in I calves with mean FG areas not different between C and I. Muscle NT content (Western blot of myofibrillar protein fraction) increased with infection; NT was immunohistochemically localized into three distinct patterns in fibers: i) sparse fiber staining, ii) dense punctuate intrafiber staining, and iii) pericystic staining. By image analysis, the greatest punctuate intrafiber pixel density of NT was associated with SO fibers from I calves with the NT colocalizing with mitochondrial Complex V - F $\mathrm{F}_{0}$ ATP synthase. More fibers were positive for the colocalization in $\mathrm{PM}$ than RF $(\mathrm{P}<0.04)$. The data are consistent with the concept that fibers rich in mitochondria possessing more inherent oxidative energy capacity generate more nitrated proteins than glycolytic fibers and as such are more affected by the proinflammatory response to infections like Sarcocystosis.

\section{Introduction}

In optimal states of good health, the inherent biological drive towards growth and maturation in the young culminates in the myriad of signals that partition a large proportion of available nutrients into tissue and bone accretion. However, disturbances to the internal environment that we call disease and stress result in some nutrients being redirected away from growth to support immune surveillance or proinflammatory protective response functions. The metabolic cost to growth for this redirection is high ${ }^{1}$ and can be observed as periods of reduced rate of growth and body weight gain. ${ }^{2}$ During the response to infection, if the diet-derived metabolic resources (energy and amino acid substrates) necessary to promote and maintain the hypermetabolic state and proinflammatory immune system upregulation become limited, the body will supply the deficit by proportionately diverting more and more nutrients away from growth as instructed through the reprioritized endocrineimmune (hormone and cytokine) signal gradient. ${ }^{3}$ Thus the need for survival overrides the drive for growth with the resulting interactions between nutrition, disease and environment shaping the needed patterns of gene expression and cellular metabolic processing. 4

Growth deficits and associated cachectic and atrophic metabolic responses (anorexia, fever production, protein and fat mobilization, liver acute phase protein synthesis) to infection in the young are evident not only in changes in body weight gain or height velocity over time but also observable as changes in body composition. ${ }^{5}$ Using models of parasitemediated progressive inflammatory disease in calves, ${ }^{6-8}$ we characterized organ and tissue depot-specific changes in chemical body composition associated with reduced rate of weight gain and/or weight loss during parasitic infection. In particular, we observed that the pattern of change in fat and protein content of impacted tissues was not uniform as regards the extent to which different tissue depots were affected. ${ }^{8}$ For example, visceral organ
Correspondence: Ted H. Elsasser,

U. S. Department of Agriculture, Bovine Functional Genomics Laboratory, Bldg 200, R. 201, B.A.R.C.-east Beltsville, MD 20705, USA.

Tel. +1.301.5048281.

E-mail: theodore.elsasser@ars.usda.gov

Key words: nitrotyrosine, muscle fiber, mitochondria, parasitic infection.

Acknowledgment: this research was conducted in part through base funding allotted to the USDA, Bovine Functional Genomics Laboratory and in part as an objective of a grant awarded to THE and JLS under the USDA NRI Competitive Grant Program.

Contributions: THE, principle investigator, concept of experiment, study design and conduct, sample collection, light microscopy, quantitative immunohistochemistry, statistical analysis, manuscript preparation; GR, sample preparation, muscle biology, statistical analysis, final manuscript approval; JE, sample preparation, muscle biology, frozen sections; SK, study conduct, sample collection, statistical analysis, final manuscript approval; AM, CF, FC, confocal microscopy; RF, parasitology; C-JL, Western and slot blot; JLS, study design, final manuscript approval.

Disclaimer: Mention of a product, reagent or source does not constitute an endorsement by the USDA to the exclusion of other products or services that perform a comparable function.

Conflict of interest: the authors report no conflicts of interest.

Received for publication: 22 March 2011. Accepted for publication: 20 April 2011.

This work is licensed under a Creative Commons Attribution 3.0 License (by-nc 3.0).

(C) Copyright T.H. Elsasser et al., 2010

Licensee PAGEPress, Italy

Veterinary Science Development 2011; 1:e3 doi:10.4081/vsd.2011.e3

weight and chemical composition were not different between infected and control animals whereas regional muscle (leg $\mathrm{v}$ back) and fat depots (visceral $\mathrm{v}$ intramuscular $\mathrm{v}$ kidneypelvic) were differentially affected. In our calf studies, particularly evident was the observation that the affected reduced muscle protein gains (actual losses in average daily protein content in some individual muscles) were more associated with what are characterized as red postural muscles than more white locomotor muscles (Bovine Mycology and Muscle Typing, http://www.bovine.unl.edu). This intrigued us for two reasons. First, the various fiber types have different metabolic characteristics and mitochondrial densities 9,10 and the mitochondrial response of a given fiber type 
may predispose that muscle to respond differently to incurred proinflammatory mediators, 11,12 largely because of the propensity of fibers higher in mitochondrial content to generate more nitric oxide (NO) and superoxide anion if perturbed by proinflammatory mediators like tumor necrosis factor- $\alpha$ (TNF- $\alpha$ ). Second, proinflammatory excesses of superoxide anion and nitric oxide might satisfy the temporal and spatial requirements needed to generate post-translational chemical protein modifications such as tyrosine nitration, detectable as 3'-nitrotyrosine (NT). ${ }^{13-16}$ Such nitrations have been further linked to polyubiquitination and overt proteosomal protein degradation. Therefore the present study was undertaken to determine whether a relationship exists between the mitochondrial content of specific muscle fiber types and the capacity for a given fiber to generate nitrated proteins and be more subject to atrophy. 16,17

\section{Materials and Methods}

\section{Experimental subjects}

The conduct of this study was approved by the USDA Beltsville Area Animal Care and Use Committee. In order to be consistent with previously-published observations on the proinflammatory immune response to Sarcocystis protozoan infection, 6,8 test subjects in the present study were castrated male (steer) Holstein calves approximately 3 -months of age and averaging $124 \pm 4 \mathrm{~kg}$ body weight at the time the infection challenge was initiated. Calves were bred and raised at the USDA Dairy Research Facility, Animal and Natural Resources Institute, at Beltsville, MD. Calves were raised initially in industry-standard outdoor calf hutches, fed a milk-replacer diet while gradually switched to a pelleted concentrate grain diet (cracked corn, soy bean meal, cotton seed hulls, vitamins and minerals; $15.1 \%$ crude protein by analysis and $2.4 \mathrm{Mcal}$ metabolizable energy/kg diet as calculated by NRC.18 All calves were treated for parasites with one application of Ivermectin ${ }^{\circledR}$ (Duravet, Blue Springs, MO, USA), and given prophylactic injections of antibiotic (Florfenicol, Nuflor ${ }^{\circledR}$, Schering-Plough, Kenilworth, NJ, USA) three weeks before infection with Sarcocystis cruzi. On the morning of the designated day of infection all animals were examined for any clinical signs infection. Rectal temperatures, white and red blood cell counts, and packed cell volumes of all animals were within normal ranges; no signs of coughing, diarrhea, nasal or eye discharge, irregular breathing, or abnormal pulmonary sounds were evident. Calves destined for infection were moved to an isolation facility and orally given a saline solution containing approxi- mately 250,000 0ocysts of Sarcocystis cruzi grown and harvested as previously described. ${ }^{6}$ After one week isolation the infected calves (INF) as well as the paired noninfected control calves (CTL) were moved to an indoor research facility and maintained in individual raised pens. The research animal housing facility was maintained at approximately $18 \pm 2{ }^{\circ} \mathrm{C}$, equipped with a constant ventilation air turnover and programmed light/dark cycles (16 $\mathrm{h}$ on and $8 \mathrm{~h}$ off). Water was provided via an automatic watering system with nipple valves and the concentrate diet was fed fresh once daily for ad libitum consumption. Feed intakes and rectal temperatures were measured daily and live body weight was obtained weekly prior to the morning feedings. All control calves were free of clinical signs of illness or distress for the duration of the pretrial 63-day experimental observation periods. The experimental growth period was defined as starting on the first day of clinical signs in INF (27 to 28 days post infection) and continuing through the following 63 days. At the conclusion of the experimental period, calves were euthanized as CTL and INF pairs on a given day. Complete dissection of the right side rectus femoris (RF) and psoas major (PM) was accomplished and representative samples prepared for either frozen sectioning for immunohistochemistry of fiber typing or immersed in $4 \%$ paraformaldehyde overnight with transfer to $70 \%$ ethanol for subsequent paraffin embedding and sectioning. Additional samples were snap frozen for further processing and use in Western blot / slot blot analysis.

\section{Metabolic fiber type and size analysis}

Initial characterizations of muscles were performed according to standard procedures defining muscle fibers according to metabolic activity criteria. Following harvest, tissue specimens approximately $8 \times 1 \times 1 \mathrm{~cm}$ were secured to thin wooden strips with string to prevent postmortem shortening, placed in sealed specimen bags, flash frozen in liquid nitrogen and subsequently transfer to $\mathrm{a}-85^{\circ} \mathrm{C}$ freezer. On the day of fiber type determination, a sample was taken from the freezer and a $1 \mathrm{~cm}$ cube cut from the specimen, which was secured to a prechilled $\left(-20^{\circ} \mathrm{C}\right)$ cryostat platform using Optimal Cutting Temperature -(OCT) TissueTek $^{\circledR}$ solution (VWR Scientific, Batavia, IL, USA). Three 8-micron sections of RF and PM for each calf were affixed to each slide. For metabolic fiber typing and morphometric sizing, slides were further processed as previously described 19,20 using the succinate dehydrogenase staining procedure followed by staining for ATPase activity after a preincubation at acid $\mathrm{pH}$ (pH 4.15). High-resolution micrographs of the stained muscle were obtained using the 40x objective containing on average 200 fibers. Fiber area was obtained using a
Zeiss Interactive Digital Analysis System (Carl Zeiss, New York, NY, USA). For statistical purposes, all fibers of a given type were counted in a photograph and from these 30 of each type were measured for area as suggested and validated previously. ${ }^{19}$ For the purposes of discussion, we equate slow oxidative (S0) fibers to what are also referred to as $\beta$-red fibers or fibers immunostaining positive for Type 1 myosin heavy chain (MHC). ${ }^{21,22}$ Similarly, fast glycolytic (FG) fibers equate to $\alpha$-white fibers immunostaining positive as Type-IIb MHC.22

\section{Isolation of myofibrillar proteins}

Samples of each muscle were pulverized to a fine powder under liquid nitrogen using a SPEX Certi-prep freezer mill (SPEX, Metuchen, $\mathrm{NJ})$. Approximately $1 \mathrm{~g}$ of pulverized tissue was homogenized (Ultra-Turrax 125 homogenizor, Janke and Kunkle GmbH, Germany) in five volumes of ice-cold pyrophosphate relaxing buffer (PRB) containing $2 \mathrm{mM} \mathrm{Na}{ }_{2} \mathrm{P}_{2} \mathrm{O}_{7}, 10 \mathrm{mM}$ Trismaleate, $100 \mathrm{mM}$ KCL, $2 \mathrm{mM} \mathrm{MgCl}_{2}, 2 \mathrm{mM}$ EGTA, $20 \mathrm{mg} / \mathrm{L}$ azide, pH 7.4, with $100 \mu \mathrm{M}$ 4-(2aminoethyl)-benzenesulfonylfluoride, HCL (AEBSF, serine protease inhibitor), $50 \mu \mathrm{M}$ pepstatin (aspartic protease inhibitor), $50 \mu \mathrm{M} \mathrm{N}$ [N-L-3-trans-carboxirane-2-carbonyl-Lleucyl]-agmatine (cysteine protease inhibitor), and $1 \mathrm{mM}$ EDTA to chelate divalent cations. The homogenate was fractionated into myofibrillar and nonmyofibrillar proteins as described previously23-25 with minor modifications. In brief, the homogenate was centrifuged at $20,000 \mathrm{x}$ g for $20 \mathrm{~min}$ at $4^{\circ} \mathrm{C}$ and the supernatant containing sarcoplasmic proteins was discarded. The pellet was rehomogenized in PRB, centrifuged, and again as described. The pellet containing myofibrillar and stromal proteins was extensively washed in five volumes of LSB buffer (PRB without $\mathrm{Na}_{2} \mathrm{P}_{27}$ ) two times in LSB with $0.5 \%$ Triton X-100 to remove a 105 to $110 \mathrm{kDa}$ sarcoplasmic reticular polypeptide and once each in LSB with $0.1 \%$ deoxycholate (DOC), in LSB alone and PRB alone, and centrifuged at $800 \mathrm{x}$ g for $10 \mathrm{~min}$ at $4^{\circ} \mathrm{C}$ between each wash. The myofibrillar proteins were then fractionated from the stromal proteins following four extractions with $0.1 \mathrm{~N}$ $\mathrm{NaOH}$ by vortexing and stirring to disrupt the pellet, followed by centrifugation at $25,000 \mathrm{x} \mathrm{g}$ for $20 \mathrm{~min}$ at $4^{\circ} \mathrm{C}$. The supernatants containing myofibrillar proteins were sonicated using two $30 \mathrm{~s}$ bursts at full power (Misonix untrasonic processor, Farmingdale, NY, USA). The amount of myofibrillar protein per gram of muscle (wet weight) was quantified using the BCA Protein Assay (Pierce Chemical, Rockford, IL, USA), with bovine serum albumin as standard.

\section{Immunohistochemistry}

Immunohistochemistry was used to localize, assess, and compare the presence of or 
changes in antigenic determinates in muscle tissue structures as affected by Sarcocystis infection. Specifically, antigens for protein nitration (NT), fiber type, and mitochondrial protein were localized in specimen sections using rabbit anti-NT (kindly donated by Dr. J. Rodrigo, Cajal Institute, Madrid, Spain), mouse monoclonal anti-myosin heavy chain F113-15F4 (Type 2a and Type 2b fibers) and anti-myosin Type 2a A4F4 (Biocytex Biotechnology, Marseille, France), ${ }^{22}$ mouse monoclonal anti-bovine Complex-V ( $\beta$-subunit $\mathrm{F}_{1} \mathrm{~F}_{0}$-ATP-synthase, Mitosciences, Inc., Eugene, OR, USA), and mouse monoclonal anti-ubiquitin (Santa Cruz Biotechnology, Santa Cruz, CA, USA), respectively. When necessary, fibers immunologically classified as FG-Type $2 \mathrm{~b}$ were differentiated in serial sections and identified by the subtractive difference between the localized total of nonstaining SO plus in the presence of fast fibers staining positive with F113-15F4 serum (Type 2a and Type 2b fibers) and those that specifically immunostain positive as Type IIa MHC with the use of antimyosin Type 2a (monoclonal antibody A4F4, Biocytex Biotechnology, Marseille, France) as previously described by Piccard et al.22 Light microscopic and tricolor confocal microscopy studies of tissues were preformed using frozen sections prepared as previously described for antigen resolution and visualization. ${ }^{26,27}$ Visualization of antigens was accomplished using the avidin-biotin-Complex (ABC) method (Vectastain elite $^{\circledR}$, Vector Laboratories, Burlingame, CA, USA) for light microscopy or goat- or donkey anti mouse- or anti-rabbit IgG labeled with either Alexa $488^{\circledR}$ or Alexa $594^{\circledR}$ (Molecular Probes Inc. Eugene, OR, USA). Frozen sections were thawed followed by a 10 min fixation in $4 \%$ freshly prepared paraformaldehyde, hydration and extensive rinsing in phosphate buffered saline. Sections were permeabilized with Triton X-100 (Sigma, St. Louis, M0, USA, 0.1 percent for 12 min), rinsed in PBS, and incubated overnight with antibodies diluted in $1 \%$ normal serum (goat or donkey) at $4{ }^{\circ} \mathrm{C}$. Fluorescent confocal images were acquired in frame mode with a Zeiss LSM 410 (Carl Zeiss, Thornwood, NY, USA) confocal microscope through a $63 \mathrm{X}$ Capochromat 1.2 numerical aperture water immersion objective. Individual optical sections for a given color spectrum were digitally recombined into a single composite (Overlay) image using LSM software (Carl Zeiss). For printing and improved visualization purposes only, both HRP-DAB and fluorescent images were enhanced in Adobe Photoshop ${ }^{\mathrm{TM}}$ as a single unitary image following compositing and flattening of the individual frames in the composite using only contrast and brightness options. In this manner, any correction associated with brightness/contrast, sharpening, or color quality was uniformly applied across all images simultaneously. Where quantitative image analysis was used to analyze images and determine a pixel density for a given immunoimage, all images captured directly from the microscope were composited with no editing or enhancement and the entire image flattened into a single master image. Quantitative image analysis of color-spectrumspecific antigen pixels (color selection established from known positive control images) was performed using Image-Pro PlusSoftware (Media Cybernetics, Silver Spring, MD) version 4.5.1. The validation criteria and procedure for the quantitative immunohistochemical assessment (image analysis of antigen pixel density was previously detailed and published. ${ }^{26-28}$ For this purpose, the master image was brought into Image-Pro ${ }^{\circledR}$ and the following sequence of steps used uniformly: a) enhance: equalize-best fit-apply; b) process: i) filters-flatten background; ii) high gause filter$7 \times 7$ pixels, 1 round at intensity $=5-$ apply; iii) pixel counting-application of established *.rge file: measurement=density sum; iv) normalization of counted pixels to counted nuclei to approximate cell numbers/field or structure; v) export data to Excel $^{\circledR}$.

\section{Slot-blot and Western blot deter- mination of protein nitration and ubiquitination}

Homogenized and extracted muscle proteins were examined for the presence of nitration and ubiquitination antigen epitopes by Western blotting procedures using anti ubiquitin and anti-NT as the primary antibodies. Samples of muscle were pulverized to a fine powder under liquid nitrogen using a SpexCertiPrep freezer mill (Model 6750, Metuchen, $\mathrm{NJ}$, USA). A portion ( $250 \mathrm{mg}$ ) of each muscle powder was placed in a $15 \mathrm{~mL}$ conical plastic tube for extraction of myofibrillar proteins. Myofibrillar proteins were extracted from muscle homogenates by traditional differential salt and pyrophosphate extraction as previously described above. Protein content of the myofibrillar extracts was determined by the $\mathrm{BCA}^{\circledR}$ protein assay (Pierce, Rockford, IL, USA) using bovine serum albumin as the standard. Extracts of the myofibrillar fraction were diluted to a standard protein concentration (10 $\mathrm{mg} / \mathrm{mL}$ ). For slot blots, $100 \mathrm{uL}$ containing $10 \mu \mathrm{g}$ total protein extracted from PM were adsorbed onto a nitrocellulose membrane matrix $(0.2 \mu$, BioRad, Richmond, CA, USA) using mild vacuum through a slot blot manifold (BioRad). For PAGE separation of proteins, $10 \mu \mathrm{g}$ aliquots of total myofibrillar protein from both $\mathrm{PM}$ and $\mathrm{RF}$ were added to $10 \mu \mathrm{L}$ SDS-loading buffer, passed through a $3 \%$ acrylamide stacking buffer and separated at $100 \mathrm{~V}$ on an $8 \%$ acrylamide gel for $1.5 \mathrm{~h}$. Proteins were transferred to nitrocellulose membranes using a BioRad semidry transfer unit. Slot blots were washed in $0.1 \%$ Tween-20 TRIS, blocked with nonfat dry milk and probed for nitrated proteins. Signals were resolved by standard chemilumenescence. For PAGE blots, nitrated protein bands were resolved using fluorescence scanning (Typhoon 9400 variable mode imager, Amersham Biosciences, Piscataway, NJ) using donkey-anti-rabbit Alexa 680 to label nitrated protein bands identified with the rabbit primary anti-NT (1:2000, kindly donated by Dr. J. P. Rodrigo, Neurosciences Division, Cajal Institute, Madrid, Spain). Slot blot, as well as Western blot, band intensities were further quantified as 0.D. units using Image-Pro ${ }^{\circledR}$ digital image analysis software (Media Cybernetics, Silver Spring, MD, USA).

\section{Statistical analysis}

Data were statistically analyzed using an analysis of variance based on the mixed models (Proc Mixed) procedure of the Statistical Analysis System. ${ }^{29}$ Main effects were designated as treatment (control vs. infected) and muscle type (RF vs $\mathrm{PM}$ ) where applicable; treatment-by-muscle was the interaction term with animal-within-treatment was used as the random error term in the model. Mean values were significantly different where $\mathrm{P}<0.05$; statistical trends were suggested where $\mathrm{P}<0.08$.

\section{Results}

In accord with the previously published timing of the onset of clinical signs of the acute phase response to schizogonous replication and redistribution of Sarcocystis throughout the body, all infected calves presented elevated temperatures $\left(>2.5^{\circ} \mathrm{C}\right.$ above normal, $\left.\mathrm{P}<0.001\right)$ on the morning of day 27 post-infection. For two weeks thereafter, voluntary feed intake was significantly lower in $S$. cruzi-infected (I) than that measured in control group (C) (mean reduction in intake $=44 \pm 7 \%, \mathrm{P}<0.03$ ). By the 4 th week post-infection mean daily ad libitum intakes of I were not significantly different from those of $\mathrm{C}$ though the mean weekly rates of weight gain has remained lower $(\mathrm{P}<0.02)$ in I as compared those calculated for C. During the overall course of the growth period, average daily body weight gain was 1.27 $\mathrm{kg} / \mathrm{d}$ for $\mathrm{C}$ and $0.83 \mathrm{~kg} / \mathrm{d}$ for I $(\mathrm{P}<0.02)$. The basic fiber type analysis of RF and PM from normal, noninfected CTL and INF as determined by traditional metabolic enzyme activity is presented in Figure 1.

Significant differences in the fiber composition of these two muscles were detected in the slow oxidative $(\mathrm{S} 0, \mathrm{P}<0.002)$ and fast glycolytic $(\mathrm{FG})$ fibers $(\mathrm{P}<0.02)$. Mean fiber types in $\mathrm{RF}$ were 11 percent S0, 38\% FOG and 51 percent FG fibers; PM consisted of 26 percent SO, 31 
percent FOG and 43 percent FG. In comparing each muscle individually between CTL and INF, there was a trend for a shift in fiber type wherein SO fiber type percent decreased from 26 to 16 percent of total fibers with a corresponding increase in fast oxidative glycolytic (F0G) fibers $(\mathrm{P}<0.1)$; a change in the percent of FG fibers as affected by infection was not evident.

The effect of end-stage Sarcocystis parasitic infection on fiber type areas is depicted in Figure 2. Infection was associated with significant reductions in fiber areas in both RF and PM for SO - Type I fibers (22\% and $49 \%$, respectively) as well as FOG - Type lla fibers in PM (58\%) with no significant change in FG Type llb fibers in either muscle, as indicative of the atrophic response to the proinflammatory challenge in these fibers. Fiber areas for Type Ila, the FOG fibers were differentially affected according to muscle as suggested by a significant infection by muscle statistical interaction $(\mathrm{P}<0.05)$; the average FOG fiber area of PM from infected calves was $58 \%(\mathrm{P}<0.02)$ smaller than similar fiber areas measured in noninfected calf PM. FOG fiber areas in RF from infected calves averaged $12 \%$ less than the areas measured in RF from noninfected calves.

In order to determine whether protein tyrosine nitration occurred in proteins isolated in myofibrillar muscle extracts changed in muscle of infected calves compared with that of muscle from healthy calves, total muscle homogenates were prepared from PM from which further was extracted a myofibrillar protein-enriched fraction. After normalizing protein content between samples, the extract was subjected to Western slot blot analysis using a rabbit polyclonal antibody to NT as the primary antibody in the blots. Figure 3 represents a slot blot resolved by chemiluminescence secondary reporter. Densitometry measurements indicated that very low levels of nitrated proteins were present in PM from CTL. When PM myofibrillar extracts were prepared from tissues obtained from INF at the end of the experimental period, slot blot analysis indicated a 14 -fold increase in NT signal $(\mathrm{P}<0.01)$ compared to that present in CTL.

Another set of myofibrillar extracts was prepared from PM and RF of both noninfected and infected calves and subjected to polyacrylamide gel electrophoretic separation to establish the pattern of nitrated proteins. When these nitrocellulose blots were further examined by densitometry image analysis, multiple protein bands for containing NT antigens were observed (Figure 4). Major bands associated with significant increases in NT 0 . D. were found at the approximate molecular weights of $200 \mathrm{kD}, 105 \mathrm{kD}$, and $44 \mathrm{kD}$. In PM extracts from some animals, additional minor lower molecular weight NT-positive bands were present throughout the lane. We did not further char- acterize these bands to determine whether these might constitute degradation products of higher molecular weight bands. Mean lane $0 . D$. increased from $0.026 \pm .003$ to $0.132 \pm .034$ $(\mathrm{P}<0.01)$ for $\mathrm{PM}$ but only increased from $.048 \pm .006$ to.061 $\pm .007(\mathrm{P}<0.06)$ in RF. The $200 \mathrm{kD}$ band intensity doubled in NT value in PM from INF as compared to CTL contemporaries, but was not different between CTL and INF calves in protein extracts from RF. The 105 $\mathrm{kD}$ band intensity increased from a mean of 0.049 0.D. units in PM from CTL to a mean of $0.31(\mathrm{P}<0.02)$ in INF and from 0.039 to 0.061 O.D. units in $\mathrm{RF}(\mathrm{P}=\mathrm{NS})$. The greatest mean NT 0.D. was associated with the $44 \mathrm{kD}$ band wherein values associated with PM samples from INF were 5 fold greater than levels measured in CTL (0.225 vs 1.01 0.D. units, $\mathrm{P}<0.04)$; The 0.D. values for NT in RF samples increased from 0.24 to $0.49(\mathrm{P}<0.05)$.

In order to refine the localization of increased NT staining as a function of fiber type, initial immunohistochemical localization of NT antigen in frozen sections of PM was performed in association with myosin heavy chain immunostaining in serial sections. As was observed in Figure 5, three distinct patterns of NT immunostaining were evident. First, an intense ring of NT was observed surrounding cysts formed at junctional boundaries of cyst and muscle fiber. The other two NT patterns were associated with specific fiber types. A light pixilated NT staining pattern was observed in PM from infected calves that corresponded to fibers in the next serial section that immunostained positive for Fast Type Ila and IIb antigen using mouse monoclonal F113-

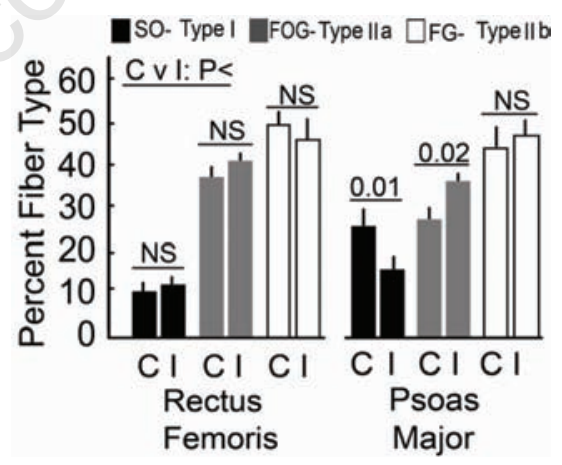

Figure 1. Representative fiber type distributions present in rectus femoris and psoas major muscles from control calves (C) and calves infected with 250,000 oocysts (I) of Sarcycystis cruzi. Designations of SO, FOG and FG represent slow oxidative, fast oxidative glycolytic, and fast glycolytis fibers, respectively, as described in association with the histochemical characteristics of the fibers described by Solomon and Dunn (1988). Solid bars represent mean values $( \pm$ S.E.M.) for 5 calves of each group.

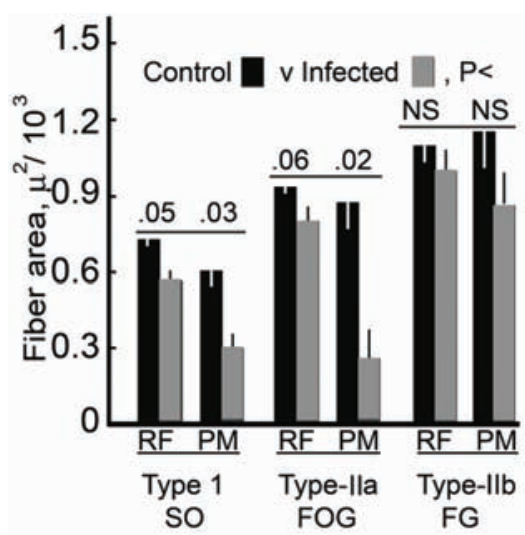

Figure 2. Fiber area analysis of rectus femoris (RF) and psoas major (PM) muscle from control and Sarcocystis-infected (250,000 oocysts per os) calves. Designation abbreviations: Type I, Type IIa, and Type IIb represent myosin heavy chain antigen reactivitiesw characteristic of SO (slow oxidative), FOG (fast oxidative glycolytic) and FG (fast glycolytic) histologic staining characteristics, respectively. Solid bars represent mean values $( \pm$ S.E.M.) for 5 calves of each group.

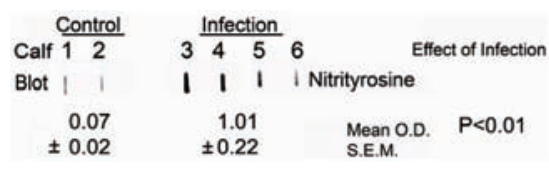

Figure 3. Slot blot analysis of myofibrillar proteins extracted from psoas major (PM) muscle homogenates of control and Sarcocystis-infected calves. Nitrotyrosine immunoreactivity in the blots was resolved using polyclonal rabbit anti-nitrotyrosine. The blot represents one replication of a series of blots. Values in the statistical analysis represent means \pm SEM for 5 calves of each infection status group.

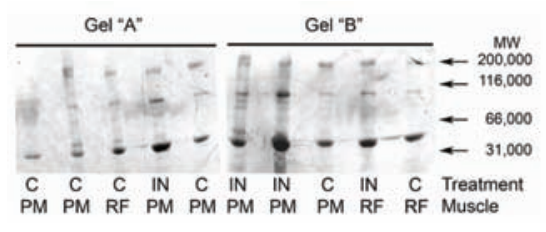

Figure 4. Representative Western blots detecting nitrotyrosine antigen immunoreactivity in myofibrillar protein extracts of rectus femoris (RF) and psoas major (PM) of control (C) and Sarcocystis-infected (IN) calves. Ten micrograms of total myofibrillar protein extract were loaded into every other lane of 10 lane gels (3\% acrylamide stacking gel on top of an $8 \%$ running gel). 
154F. In the majority of positive staining fibers of this character, the NT immunostaining was most apparent in the outer areas of the fiber in close approximation with the border of another fiber. The greater intensity of NT immunostaining was evident in serial section fibers not staining with the F113-15F4 and therefore considered slow twitch Type 1 fibers; this denser pixilation was distributed throughout the slow fibers. There was little evidence of an effect of infection to change levels of NT immunostaining in connective tissue, while a very low level of NT was detectable in some vascular endothelial cells and infiltrating monocytes in both muscle and connective tissue.

Tricolor confocal fluorescence microscopy studies of both RF and PM (Figure 6 - panels A and $\mathrm{B}$ ) confirmed this pattern of fiber-specific NT immunostaining and extended the observations and comparisons made in the serial section studies represented by Figure 5 . Here we demonstrate that the slow fibers in RF similarly responded to infection with higher increases in NT as compared to that which developed in fast Type IIa and llb fibers (Panel A). When fibers were differentiated on the basis of myosin heavy chain immunostaining in PM (Panel B) and respective fibers measured, image analysis calculations demonstrated that the mean fiber areas of Type I fibers was approximately 32 percent smaller in INF than Type 1 fibers in CTL. Similarly, where the presence of NT immunostaining was strongly present in PM from INF, NT staining in muscle from CTL was hardly detectable at all. Across fibers from both RF and PM, image analysis revealed an overall mean NT immunostaining pixel level more than 5-fold higher in fibers that did not immunostain (therefore corresponding to S0 fibers) with the F113-15F4 antibody as compared to the pixel density measured in F113-15F4-positive (FOG + FG) staining fibers (bottom panel; 1035 \pm 181 v 194 \pm 40 pixels, $\mathrm{P}<0.02$ ). Finally, overlay images generated with the combination of anti-NT and anti-Complex $\mathrm{V}$ sera resolved and confirmed the colocalization of NT with the mitochondria ATP-Synthase protein - (F1F0-Complex V, Panel C).

\section{Discussion}

To the best of our present knowledge, the present findings are the first to demonstrate the involvement of selective protein tyrosine nitration as a mechanism of pathogenesis in muscle during the chronic phase of this parasitic infection. Sarcocystis is a member of a larger family of related cyst-forming coccidialike parasites including Neospora and Toxoplasma that are largely ubiquitous throughout the world. Diagnosed infections and outbreaks have documented a variety of pathobiological issues ranging from pregnancy complications and neonatal death in humans as well as livestock, companion animals and wild animals to growth and metabolic perturbations. 30,31 The early onset infection process is highly proinflammatory even in low-level infections. ${ }^{31}$ In its chronic state, Sarcocystis infection can serve to prime immunological responses in a manner where proinflammatory responses elicited by other agents (Gramnegative bacterial lipopolysaccharide, for example) are exacerbated and cause more severe pathological consequences. ${ }^{32}$ These previous observations are in line with the present data wherein a rather constitutive upregu- lation in pathway responses occurs to generate the required reactive nitration species. From an experimental standpoint, Sarcocystis cruzi infection is an interesting model in which to study the proinflammatory response because the timing of biological life cycle events of the Sarcocystis organism in the progression from vascular endothelial eruption to muscle cyst formation is relatively precise and permits researchers to study immune system and homeostatic responses specific to different aspects or stages of the proinflammatory response. 33,34

Because of the rapidity of onset and severity of the robust clinical signs distress, much attention has been focused on the events sur-

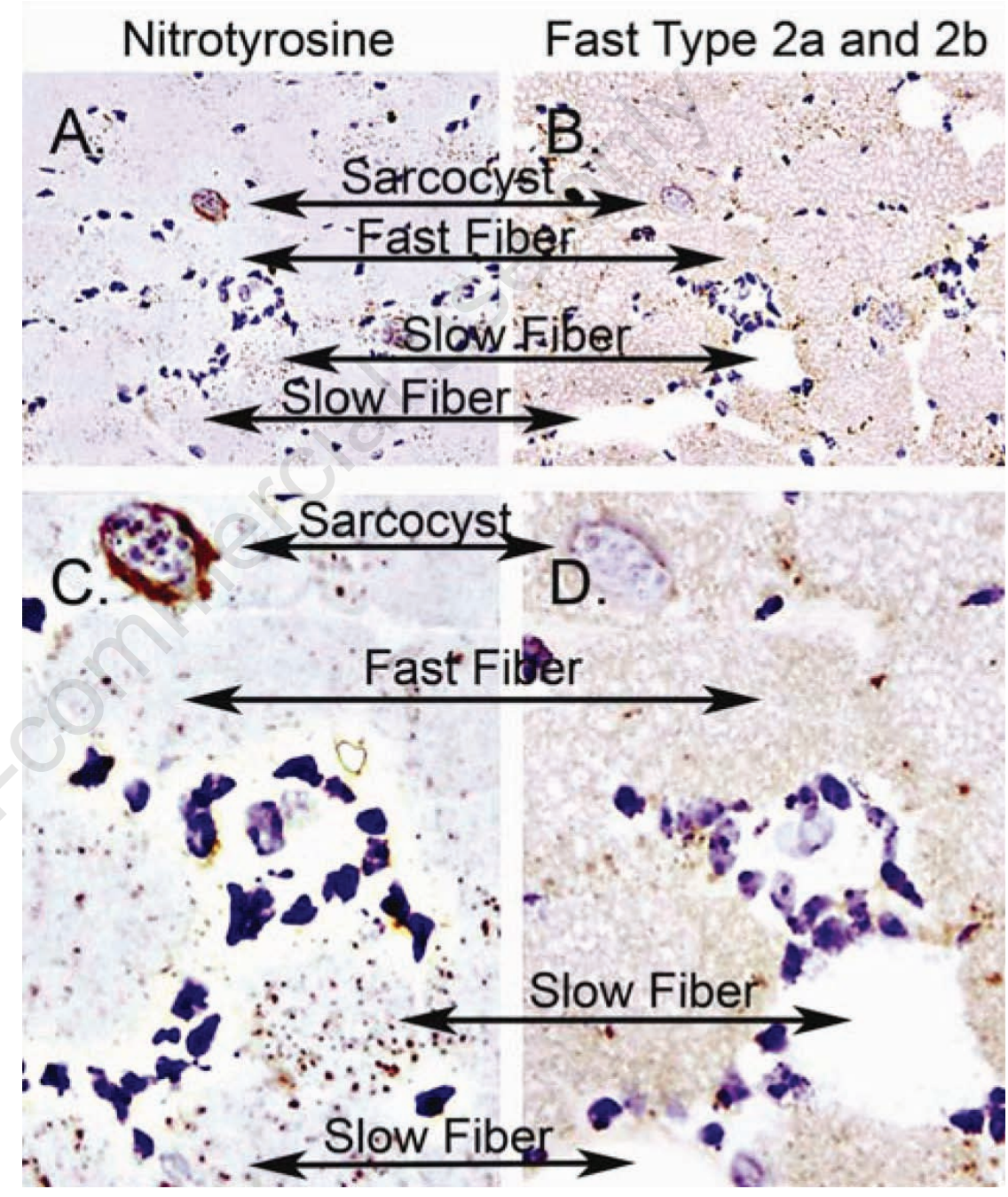

Figure 5. Immunohistochemical localization of nitrotyrosine antigen (Panels A, C) and fiber type localization (Panels B, D) in psoas major muscle from a calf infected with Sarcocystis cruzi. Antigen localization was accomplished using anti-nitrotyrosine $(1: 5000)$ and an antibody that recognized both fast myosin heavy chain IIa and IIb antibody (F113-15F4). Antigens were and visualized using avidin-biotin conjugated (ABC) horseradish peroxidase and diaminobenzadine as the enzyme substrate. The brown color represents positive immunostaining for a respective antigen as labeled; nuclei were counterstained in blue Curazzi's hematoxylin. Slow (Type I) fibers did not react with the fast myosin heavy chain anti Type IIa,b antibody and are indicated as clear areas in the right panels. Panels $\mathrm{C}$ and $\mathrm{D}$ are $5 \mathrm{x}$ magnifications of image regions as indicated in Panels $\mathrm{A}$ and $B$, respectively. 

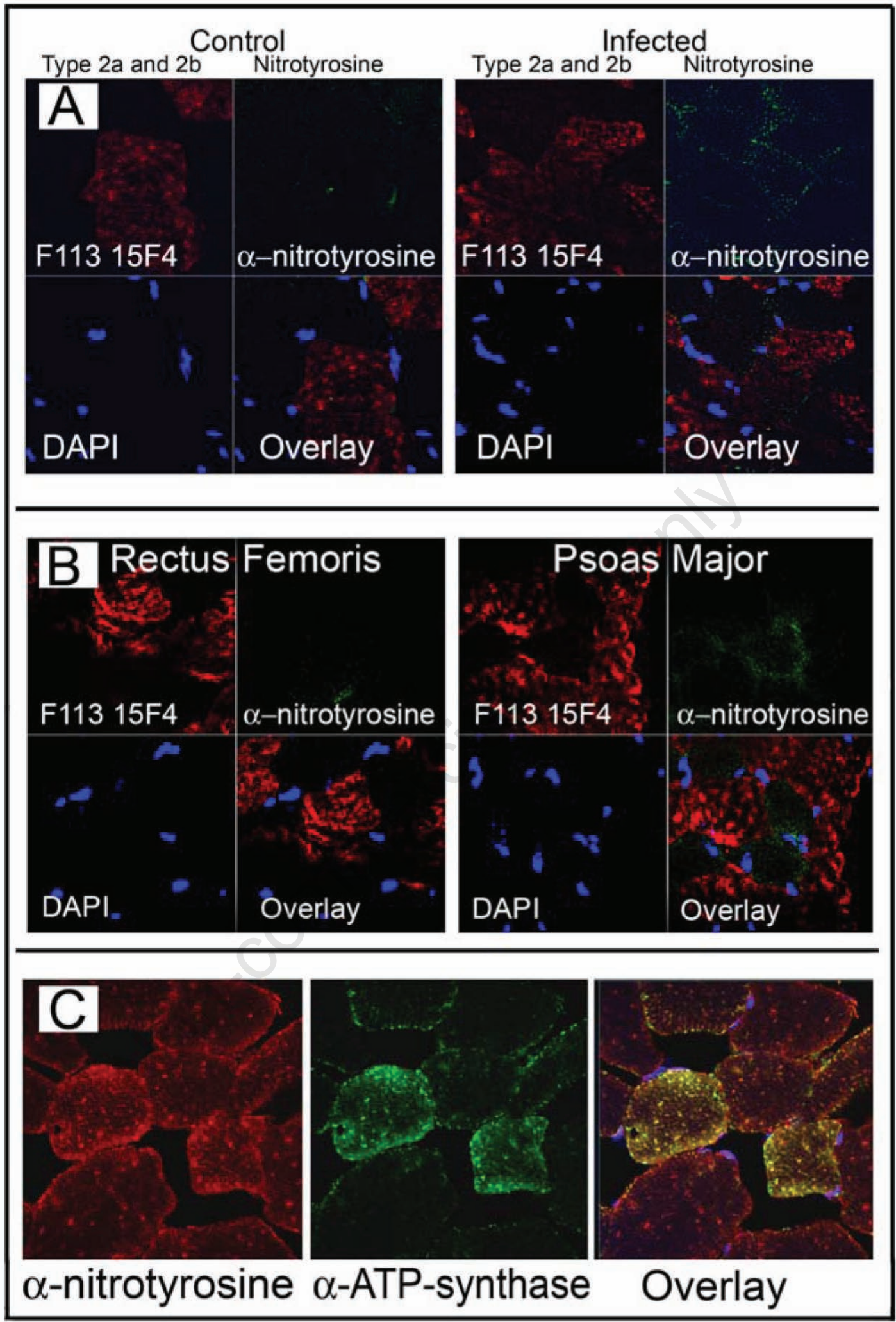

Figure 6. Confocal fluorescence imaging of nitrotyrosine and fiber type in muscle from control and Sarcocystis-infected calves. Top Panel: as represented in sections of psoas muscle from control and infected calves, nitrotyrosine antigen $(\alpha$-nitrotyrosine) appears as green pixels, where red pixels represent Type IIa and llb (antibody F113 15F4) muscle fibers, and blue represents DAPI-stained nuclei. Slow oxidative Type-1 fibers were represented by fibers devoid of red pixels. Fluorescent green pixels were minimally present in any fiber type in psoas major of control calves while a significant increase in nitrotyrosine pixels is apparent in several fibers of PM from an infected calf. Bottom Panel: a representative comparison of fiber-specific nitrotyrosine immunoreactivity in both rectus femoris and psoas major from an infected calf indicated that the majority of nitrotyrosine pixels as measured by color-specific image analysis colocalized with fibers of smaller cross sectional area, with the greatest nitrotyrosine pixel density localized in the smallest fibers which did not stain fluorescent red for Fast Type IIa,b antigenicity. 
rounding the acute phase response associated with the abrupt Sarcocystis outburst on day 27 post-infection where among other pathological features significant vascular endothelial disruption is evident. ${ }^{34}$ In the present paper, we chose to investigate some of the mechanisms underlying the persistent stunting, growth deficits and prolonged catabolic loss that is characteristic of the later stages of infection where clinical signs of sickness are not readily apparent though animals still fail to thrive.

At 63 days after infection, sarcocysts were detected in skeletal muscle with appearances similar to those described previously. ${ }^{35,36}$ Interestingly, at this point in the infection voluntary feed intake of infected calves was not significantly different from that of noninfected calves though the rates of gain of the infected calves were substantively depressed relative to the mean rate of live weight gain of their healthy cohorts (data not presented). The cysts were irregularly shaped containing out-swellings demonstrating the presence of the contained metrocytes. All cysts observable in both rectus femoris and psoas major muscle sections immunostained positive for NT with a pattern emanating in a gradient manner from most intense at the interface between the cyst and host muscle to least detectable approximately 100 to 150 microns away from the cyst. Larger cysts containing higher numbers of metrocysts appeared to have the greater diameter of pericystic positive immunostaining for NT.

Confocal fluorescent microscopy confirmed data from Western blot analysis initially indicating that the muscle content of nitrated proteins were increased in muscle from infected calves compared to the content of these antigenic determinates in normal animals. Microscopic analysis from both light microscopy of serial muscle sections as well as confocal localization of fluorescent labeled antigen signals indicated that the pattern of nitration was greater in slow-twitch Type I myosin heavy chain fibers than fast-twitch Type II fibers, Type IIb fibers having the lowest levels of nitration in infected calf muscle. Though it was beyond the goal of this study to pursue the point, it would appear that the increased levels of nitration present in slow twitch-Type I fibers could be a signal for ubiquitination of proteins associated with these fibers resulting in more intracellular protein degradation and therefore the smaller crosssectional area of these fibers, as previously suggested by Souza et al.16,37 in other proinflammatory nitration events.

The impact of mitochondria-derived free radical damage to cells and muscle fibers has been demonstrated previously in association with over production of peroxynitrite (ONOO-) via mitochondrial nitric oxide synthase ${ }^{38}$ and muscle nuclear apoptosis where TNF- $\alpha$-driven overactivation of the nitric oxide cascade pro- motes free radical leakage. ${ }^{39}$ It is important to remember, it was at this 63 day point after oral infection that previously we had measured increased plasma levels of TNF- $\alpha$ in Sarcocystis-infected calves ${ }^{40,41}$ consistent with the observations by Fayer et al. ${ }^{36}$ that indicated that extracts of Sarcocystis bradyzoites were capable of stimulating macrophage secretion of TNF- $\alpha$ in vitro. The present findings extend these observations of TNF- $\alpha$ and support the concept that components of the proinflammatory cascade downstream of TNF- $\alpha$ action are elicited locally in muscle that involve the generation of endogenous reactants capable of nitrating tyrosine residues of myofibrillar proteins. Similarly, the present findings provide a molecular explanation of previous observations from our laboratory wherein it was shown for the first time that the growth retardation associated with the proinflammatory impact of protozoan parasitic infection in Holstein calves was significantly more evident in red postural muscle (such as psoas major) than locomotor muscles (as typified by rectus femoris). Furthermore, it was demonstrated in our previous study that the observed muscle effects exceeded any of the effects associated with the temporary slump in voluntary feed intake where direct nutritional effects of nutritional plane were addressed statistically through the use of the applied pair-feeding strategy. ${ }^{7}$ Protein tyrosine nitration is a posttranslational chemical modification wherein the 3'-position of the affected tyrosine phenolic structure becomes nitrated. 42 Though thought of principally as a biomarker for oxynitrogen stress and disease, ${ }^{43}$ we now know that nitration in low, well controlled biological circumstances such as the selective nitration of JAK2 kinase by growth hormone ${ }^{27}$ functions as a process important to signal transduction events, possible countering hormone and cytokine pathway processes activated by phosphorylation while the high levels of production indicate pathology. ${ }^{28,43}$ While some speculation exists regarding the source of the nitrating reactant species, a large body of evidence suggests that the main nitrating agent is peroxynitrite $\left(\mathrm{ONOO}^{-}\right)$, a potent reactant formed in the localized reaction between nitric oxide and superoxide anion. ${ }^{42,43}$ Though short-lived in biological half-life $\mathrm{ONOO}^{-}$in muscle appears to be formed in close approximation to dysfunctional mitochondria where the generation of superoxide anion is excessive. 44 The need for spatial and temporal co-generation of superoxide anion and NO to produce $\mathrm{ONOO}^{-}$is capable of being satisfied within the mitochondria per se. Recent data indicate that in response to proinflammatory mediators like TNF- $\alpha$, mitochondria generate excess superoxide anion as the electron transport chain progressively deteriorates, nitric oxide is generated through mNOS, and signaling occurs through NO-modifications to proteins like cytochrome-C oxidase and guanylate cyclase with many of these reactions setting the stage for apoptotic responses. ${ }^{45-50}$ For the most part, the physiological functionality and metabolic character of individual muscles is associated with the ATP-generating capacity of the muscle fibers and the nature of energy production (oxidative phosphorylation vs. glycolysis). Specifically then, this points to the mitochondrial makeup as well as the expression and activity of various isoforms of nitric oxide synthase of muscle fibers as factors underlying the observed predominant impact of Sarcocystis infection on muscle fibers largely functioning through oxidative metabolism. Older data recognized that certain myopathies associated with endocrine dysfunction affecting carbohydrate and protein metabolism (Cushings disease, thyroiditis, diabetes) specifically affected Type II muscle fibers. ${ }^{51}$ More recent investigations suggest that the catabolic response of muscle to a given pathological stimulus as a function of fiber type involvement is complex. Where the specificity of impact of disease processes was examined in regard to affected fiber types, Li et al. 52 indicated that fast glycolytic Type IIb fibers were selectively atrophied in skeletal muscle of patients with chronic heart failure. This response also was observed in studies where subjects with left ventricular dysfunction presented Type IIb-specific fiber atrophy. ${ }^{11}$ However, the proinflammatory stress of septicemia as directed through cytokine mediators like TNF- $\alpha$ appears to trigger responses more akin to those we observed here where concomitant with increased proteosomal activity the predominant impact on muscle fiber atrophy was specifically associated with slow oxidative-Type I fibers. ${ }^{12}$ The present data are therefore consistent with the concept that cytokine-mediated proinflammatory stress induces selective nitration as well as ubiquitination of proteins directing the involved proteins towards elimination from the cell. Collectively, the present data reflect and further underscores the diagnostic value of fiberspecific nitration patterns as a biomarker of selective protein degradation in specific fiber types ${ }^{53-57}$ and the further capacity to suggest appropriate interventions to reign-in aberrant muscle degradation.

Given that there is no apparent preference for what fiber types are infected by the Sarcocystis cruzi organism itself, the selective effect on the slow fibers is consistent with the premise that the response may be more a function of the metabolic and/or mitochondrial makeup of the affected fibers. ${ }^{21,22,58} \mathrm{~A}$ unique finding of the present research is the identification of increased fiber-specific NT immunostaining patterns closely approximating the mitochondrial staining patterns observed in 
traditional ATPase fiber differentiation here represented by the colocalization of NT to the mitochondrial $\mathrm{F}_{1} \mathrm{~F}_{0}$-ATP synthase on Complex V. Furthermore, the fact that fiber types differentially express various isoforms of nitric oxide synthase may also contribute to the selective impact of the slow fibers in our study 59 though this is a feature of our model that we have yet to verify. The significant question remains, however, as to whether the presence of these NT-associated SO fiber catabolic events constitute an attempt on the part of the host to increase its chances for survival or simply reflect the mechanism through which the catabolic events continue to unfold in the chronic Sarcocystis-infected animal.

\section{References}

1. McDade TW. Life history, maintenance, and the early origins of immune function. Am J Human Biol 2005; 17:81-94.

2. Stephensen CB. Burden of Infection on Growth Failure. J Nutr 1999;129:534-8S.

3. Elsasser TH, Klasing KC, Filipov N, et al. The metabolic consequences of Stress: Targets for stress and priorities of nutrient use. In: Moberg, G. and Mench, J., eds., in The Biology of Animal Stress: Basic Principles and Implications for Animal Welfare. 2000; CABI Publishing, New York, NY, USA, pp. 77-110.

4. Paoloni-Giacobino A, Grimble R, Pichard C. Genomic interactions with disease and nutrition. Clin Nutr 2003; 22:507-14.

5. Jacobi SK, Gabler NK, Ajuwon KM, et al. Adipocytes, myofibers, and cytokine biology: new horizons in the regulation of growth and body composition. J Anim Sci 2006; 84 Suppl:E140-9.

6. Elsasser TH, Hammond AC, Rumsey TS, et al. Perturbed metabolism and hormonal profiles in steers infected with Sarcocystis cruzi. Dom Anim Endocrinol 1986;3:27787.

7. Elsasser TH, Rumsey TS, Hammond AC, et al. Influence of parasitism on plasma concentrations of growth hormone, somatomedin-C and somatomedin-binding proteins in calves. $\mathrm{J}$ Endocrinol 1988;116:191-200.

8. Elsasser TH, Sartin JL, McMahon C, et al. Changes in somatotropic axis response and body composition during growth hormone administration in progressive cachectic parasitism. Dom Anim Endocrinol 1998;15:239-255.

9. Gueguen N, Lefaucheur L, Fillaut M, et al. Muscle fiber contractile type influences the regulation of mitochondrial function. Mol Cell Biochem 2005;276:15-20.

10. Gueguen N, Lefaucheur L, Fillaut M, et al.
Control of skeletal muscle mitochondria respiration by adenine nucleotides: differential effect of ADP and ATP according to muscle contractile type in pigs. Comp Biochem Physiol 2005;140:287-297.

11. Delp MD, Duan C, Mattson JP, et al. Changes in skeletal muscle biochemistry and histology relative to fiber type in rats with heart failure. J Appl Physiol 1997; 83:1291-9.

12. Minnaard R, Drost MR, Wagenmakers AJ, et al. Skeletal Muscle wasting and contractile performance in septic rats. Muscle Nerve 2005; 31:339-348.

13. Goss SP, Singh RJ, Hogg N, et al. Reactions of *NO, *NO2 and peroxynitrite in membranes: physiological implications. Free Radic Res 1999; 31:597-606.

14. Goligorsky MS, Hong L, Brodsky S, et al. Relationships between caveolae and eNOS: everything in proximity and proximity in everything. Am J Physiol Renal Physiol 2002;283:1-10.

15. Heijnen HF, van Donselaar E, Slot JW, et al. Subcellular localization of tyrosine-nitrated proteins is dictated by reactive oxygen species generating enzymes and by proximity to nitric oxide synthase. Free Radic Biol Med. 2006;40:1903-13.

16. Souza JM, Choi I, Chen Q, et al. Proteolytic Degradation of Tyrosine Nitrated Proteins. Arch Bioch Biophys 2000; 380:360-6.

17. Paoloni-Giacobino A, Grimble $\mathrm{R}$, and Pichard C. Genomic interactions with disease and nutrition. Clin Nutr 2003;22:50714.

18. NRC, National Research Council. Nutrient Requirements of Beef Cattle, National Academy Press, Washington, DC, USA, 1984.

19. Solomon MB, Dunn MC. Simultaneous histochemical determination of three fiber types in single sections of ovine, bovine and porcine skeletal muscle. J Anim Sci 1988;66:255-64.

20. Ono Y, Solomon MB, Elsasser TH. Effects of Synovex-S and recombinant bovine growth hormone (Somavubove) on growth responses of steers: II. Muscle morphology and proximate composition of muscles. $\mathrm{J}$ Anim Sci 1996;74:2929-34.

21. Pearson AM, Young RB. Skeletal muscle fiber types. In: Muscle and Meat Biochemistry. Academic Press, New York, NY, USA, 1989, pp. 235-265.

22. Picard B, Duris MP, Jurie C. Classification of bovine muscle fibers by different histochemical techniques. Histochem $\mathrm{J}$ 1998;30:473-9.

23. Tiao G, Fagan J, Roegner V, et al. Energyubiquitin-dependent muscle proteolysis during sepsis in rats is regulated by glucocorticoids. J Clin Invest 1996;97:339-348.

24. Jaspers SR, Fagan JM, Satarug S, et al.
Effects of immobilization on rat hind limb muscle under non-weight-bearing conditions. Muscle Nerve 1988;11:458-466.

25. Etlinger JD, Zak R, Frischman, DA. Compositional studies of myofibrills from striated muscle. J Cell Biol 1976;68:123-41.

26. Elsasser TH, Kahl S, MacLeod C, et al. Mechanisms underlying growth hormone effects in augmenting nitric oxide production and protein tyrosine nitration during endotoxin challenge. Endocrinology 2004;145:3413-23.

27. Elsasser TH, Li CJ, Caperna TJ, et al. Growth hormone (GH)-associated nitration of Janus kinase-2 at the 1007Y-1008Y epitope impedes phosphorylation at this site: mechanism for and impact of a $\mathrm{GH}$, $\mathrm{AKT}$, and nitric oxide synthase axis on $\mathrm{GH}$ signal transduction. Endocrinology 2007;148:3792-802.

28. Elsasser TH, Kahl S, Li CJ, et al. Caveolae nitration of Janus kinase-2 at the 1007Y$1008 Y$ site: coordinating inflammatory response and metabolic hormone readjustment within the somatotropic axis. Endocrinology 2007;148: 3803-13.

29. SAS. SAS/STAT Software, Changes and enhancements through version 6.11. 1996. SAS Institute, Cary, NC, USA, pp. 589-625.

30. Daugschies A, Hintz J, Henning M, et al. Growth performance, meat quality and activities of glycolytic enzymes in the blood and muscle tissue of calves infected with Sarcocystis cruzi. Vet Parasitol 2000;88:7-16.

31. Dubey JP, Lindsay D. Neosporosis, toxoplasmosis, and sarcocystosis in ruminants. Vet Clin North Am Food Anim Prac 2006;22:645-71.

32. Elsasser TH, Sartin JL, Martinez A, et al. Underlying disease stress augments plasma and tissue adrenomedullin (AM) responses to endotoxin: colocalized increases in AM and inducible nitric oxide synthase within pancreatic islets. Endocrinology 1999;140:5402-11.

33. Johnson AJ, Hildebrandt PK, Fayer R. Experimentally induced Sarcocystis infection in calves: pathology. Am J Vet Res 1975;36:995-9.

34. Fayer, R, Elsasser TH. Bovine Sarcocystis: How parasites negatively affect growth. Parasitology Today 1991;7:250-5.

35. Pacheco ND, Sheffield HG, Fayer R. Fine structure of immature cysts of Sarcocystis cruzi. J Parasitol 1978;64:320-5.

36. Fayer R, Andrews C, Dubey JP. Lysates of Sarcocystis cruzi bradyzoites stimulate RAW 264.7 macrophages to produce tumor necrosis factor (cachectin). J Parasitol 1988;74:660-4.

37. Abello N, Kerstjens HA, Postma DS, et al. Protein tyrosine nitration: selectivity, physicochemical and biological conse- 
quences, denitration and proteomics methods for the identification of tyrosinenitrated proteins. J Proteome Res 2009;8:3222-8.

38. Boveris A, Alvarez S, Navarro A. The role of mitochondrial nitric oxide synthase in inflammation and septic shock. Free Radic Biol Med 2002;33:1186-93.

39. Li YP, Reid MB. NF-kappaB mediates the protein loss induced by TNF- $\alpha$ in differentiated skeletal muscle myotubes. Am J Physiol Regul Integr Comp Physiol 2000;279:R1165-70.

40. Kenison DC, Elsasser TH, Fayer R. Radioimmunoassay for tumor necrosis factor- $\alpha$ : Concentrations and circulating molecular forms. J Immunoassay 1990;11:177-98.

41. Kenison DC, Elsasser TH, Fayer R. Tumor necrosis factor as a potential mediator of acute metabolic and hormonal responses to endotoxemia in calves. Am J Vet Res 1991;52:1320-26.

42. Greenacre SA, Ischiropoulos H. Tyrosine nitration: localization, quantification, and consequences for protein function and signal transduction. Free Radic Res 2001;34:541-581.

43. Szabó C, Ischiropoulos H, Radi R. Peroxynitrite: biochemistry, pathophysiology and development of therapeutics. Nat Rev Drug Discov 2007;6:662-80.

44. Boczkowski J, Lisdero CL, Lanone S, et al.
Endogenous peroxynitrite mediates mitochondrial dysfunction in rat diaphragm during endotoxemia. FASEB J 1999;13:1637-46.

45. Afanas'ev IB. Signaling functions of free radicals superoxide and nitric oxide under physiological and pathological conditions. Mol Biotechnol 2007;37:2-4.

46. Moncada S, Bolaños JP. Nitric oxide, cell bioenergetics and neurodegeneration. $\mathrm{J}$ Neurochem 2006;97:1676-89.

47. Ghafourifar P, Asbury ML, Joshi SS, Kincaid ED. Determination of mitochondrial nitric oxide synthase activity. Methods Enzymol 2005;396:424-44.

48. Clementi E., Nisoli E. Nitric oxide and mitochondrial biogenesis: a key to longterm regulation of cellular metabolism. Comp Bioch Physiol and Mol Integr Physiol 2005;142:102-105.

49. Carreras M, Franco MC, Peralta JG, et al. Nitric oxide, complex I, and the modulation of mitochondrial reactive species in biology and disease. Mol Aspects Med 2004;25:125-139.

50. Chande NS, Trzyna WC, McClintock DS, et al. Role of oxidants in NF-kappa B activation and TNF-alpha gene transcription induced by hypoxia and endotoxin. J Immunology 2000;165:1013-21.

51. Ruff RL, Weissmann J. Endocrine myopathies. Neurol Clin 1988; 6:575-592. 52. Li P, Waters RE, Redfern SI,et al.
Oxidative phenotype protects myofibers from pathological insults induced by chronic heart failure in mice. Am J Pathol 2007;170:599-608.

53. Mitch WE, Goldberg AL. Mechanisms of muscle wasting. The role of the ubiquitinproteasome pathway. N Engl J Med 1996;335:1897-905.

54. Schwartz AL, Ciechanover A. The ubiquitin-proteasome pathway and pathogenesis of human diseases. Ann Rev Med 1999;50: 57-74.

55. Hershko A, Ciechanover A, Varshavsky A. Basic medical research award: the ubiquitin system. Nat Med 2000;6:1073-81.

56. Yi D, Perkins PD. Identification of ubiquitin nitration and oxidation using a liquid chromatography/mass selective detector system. J Biomol Tech 2005;16:364-70.

57. Bar-Shai M ,Reznick AZ. Reactive nitrogen species induce nuclear factor-kappa Bmediated protein degradation in skeletal muscle cells. Free Radic Biol Med 2006;40:2112-25.

58. Peter JB, Barnard RJ, Edgerton VR, et al. Metabolic profiles of three fiber types of skeletal muscle in guinea pigs and rabbits. Biochemistry 1972;1:2627-33.

59. Punkt K, Fritzsche M, Stockmar C, et al. Nitric oxide synthase in human skeletal muscles related to defined fiber types. Histochem Cell Biol 2005;125:567-73. 